\title{
Coopetition Spectrum Trading - Creating Endogenous Spectrum Holes
}

\author{
Liu Cui \\ School of Information Sciences \\ University of Pittsburgh \\ Email: lic49@pitt.edu
}

\author{
Taieb Znati \\ Department of Computer Sciences \\ University of Pittsburgh \\ Email: znati@pitt.edu
}

\author{
Martin B.H. Weiss \\ School of Information Sciences \\ University of Pittsburgh \\ Email: mbhw@pitt.edu
}

\begin{abstract}
Dynamic spectrum access is an important way to alleviate the spectrum scarcity. Traditionally, secondary users are allowed to opportunistically operate when primary users are absent and buy idle bands from primary users. Although it improves the spectrum utility to some extents, it is not enough. In this paper, we argue that spectrum hole should be endogenous which means they should be negotiated by primary and secondary users. We proposed a novel spectrum sharing scheme with spectrum usage and management right. Further, we suggest spectrum trading as a financial option to capture the realistic usage and increase the trading flexibility. We show the superior of our model for primary users in deterministic and dynamic leasing environments.
\end{abstract}

\section{INTRODUCTION}

The rapid proliferation of various forms of mobile devices, coupled with the expansion of wireless Internet services, brought to the forefront the limitations of the static command and control approach to spectrum management. Exclusive access to rigidly partitioned bands of the spectrum has succeeded in ensuring predictable, interference-free access to the wireless medium. The licensing of the spectrum bands for extended periods of time over large geographical areas, however, resulted in unlicensed bands being severely congested, while large portions of licensed spectrum lie unused, despite the soaring demand for wireless broadband. To address the apparent spectrum scarcity, dynamic spectrum allocation (DSA) was proposed as an alternative to fixed allocation. DSA enables dynamic spectrum sharing, in time and space, thereby providing the flexibility needed to respond to temporal and spatial variations of traffic statistics and bandwidth requirements of different services [1].

Two types of spectrum sharing schemes exist in DSA, opportunistic and cooperative spectrum sharing. Opportunistic sharing that enabled by sensing and programmable transmitters was the dominant approach in the past. The intense research in opportunistic sharing has led to several schemes for efficient spectrum management and allocation. However, most of them use questionable assumptions that limit their applicability in a real settings. For example, spectrum holes are considered to be exogenous, assumed to occur through some phenomena the secondary users (SUs) can neither control or anticipate. Furthermore, spectrum exogeneity assumes that primary users' (PUs) behavior is invariant to the presence of SUs. This may be an unrealistic assumption [2].
An alternative to opportunistic sharing is the more economically-centric approach, cooperative sharing. The kernel of cooperative sharing is explicit bargaining over spectrum usage rights, e.g. spectrum trading in the secondary spectrum market. The majority of research work in spectrum trading uses game theoretic and auction based approaches. Game theoretic approaches are targeted at finding the best strategies (Nash equilibrium or Pareto Optimality) that maximize the utilities of PUs and SUs in different scenarios [3] [4] [5]. Auction based research views spectrum as a divisible good and studies auction mechanisms to allocate these goods to achieve an optimal allocation. "Optimal" may imply optimality for PUs, SUs, and social welfare [6] [7]. The major limitation for this research is that they assume spectrum trading only takes place on PUs' idle bands. In other words, although SUs have higher QoS and PUs earn revenue through trading, SUs' still do not have control of the spectrum holes. Moreover, these schemes do not provide enough flexibility to manage risk while creating incentives.

In cooperative sharing, spectrum holes are explicitly endogenous. These holes are created as the result of bilateral bargaining on the part PUs and SUs, or they may occur through a broker or a transaction in a spectrum market. It is often the case, however, due to the variability of user traffic load and requirements, that it is difficult for PUs to accurately estimate the amount of endogenous spectrum that could be leased to SUs, without inadvertently limiting their ability to meet future needs. This, in turn, leads to conservative PU leasing strategies, thereby reducing the amount of leasable spectrum.

To address these shortcomings and challenges, we propose a framework for coopetition to foster collaboration among PUs and SUs, while allowing competition. The coopetition framework ensures the spectrum is allocated to those who value it the most. In order to achieve this goal, spectrum bands are separated into two different blocks, called spectrum management right and spectrum usage right. When SUs buy the management right, they fully control the spectrum for the entire period of time. On the other hand, if SUs buy spectrum usage right, they have to follow PUs' sharing etiquette and PUs have the right to revoke the spectrum if needed. Both rights are traded as financial options. More specifically, our framework ensures the following: 
1) creation of endogenous spectrum holes to augment those vacated when PUs become idle;

2) combine exogenous and endogenous spectrum holes and provide opportunities for spectrum usage that go beyond what is currently possible through current spectrum sharing schemes;

3) incentivize PUs to engage an measured risk taking spectrum sharing approach while offering them opportunities to revoke spectrum when needed.

4) support put and call options to provide flexibility to PUs and SUs in trading endogenous spectrum holes to achieve effective spectrum sharing.

Two cases are analyzed in this paper, static spectrum allocation and dynamic spectrum allocation. It is shown that the proposed model provides at least the same profits as the current spectrum trading model where PUs only sell idle bands and it is superior in most of the cases. Three types of risk functions and two types of penalty functions are implemented for sensitivity analysis. Spectrum strategies that lead to higher profits are determined for each case as well.

To the best of our knowledge, the proposed coopetition framework is the first model that allows PUs and SUs to cooperate with each other while compete in the same group. The endogenous spectrum hole that created by this model will benefit spectrum trading and utilization, since it offers PUs a chance to compare the gain via spectrum leasing and their own services. It also provides SUs an opportunity to demand exactly what they want. It is likely that these two aspects will increase the market liquidity to some extent, though we do not study that here. An additional contribution of htis paper is to allow the trading of different spectrum rights and allow the revocation of usage rights, which mitigates the risks of spectrum trading and should increase the tradeable channels.

The rest of the paper is organized as follows. Section II describes the coopetition framework we proposed. Section III is the system model with utility functions. Section IV provides evaluations. Finally, Section V concludes the paper and discusses future research.

\section{COOPETITION FRAMEWORK}

Without loss of generality, we assume that at any point of time, PUs have a minimum requirement for the number of wireless channels they need to provide services and additional channels for potential demand. PUs should be encouraged to create more (endogenous) spectrum holes since it brings higher spectrum leasing fees for PUs and more spectrum access opportunities for SUs. Though it optimizes spectrum utilization, there are risks associated with this action as well. For example, extra service demand that may happen in the future cannot be predicted now. In order to mitigate the potential risks and uncertainties, we propose a novel spectrum sharing structure with two types of spectrum rights: a spectrum usage right and a spectrum management right.

As shown in Fig.1, the entire licensed band for $\mathrm{s} P U$ is separated into three parts: exclusive usage for PUs $(E)$, spectrum usage right for SUs $(U)$, and spectrum management right for SUs $(M) . E$ is reserved for the PU's wireless services and can not be accessed by any other spectrum users. The PU gives up spectrum management rights in $M$, so the SUs in $M$ have full control of the resource. They can allow other spectrum users to access based on the spectrum sharing etiquette they designed. This works the same as when PUs only sell idle bands. The risk taking is embodied in spectrum usage right. In $U$, PUs still have the right of management and SUs have the right to operate in accordance with the PU's requirements. Basically, PUs have two actions in $U$. They can share the spectrum with SUs or revoke the spectrum from SUs' usage.

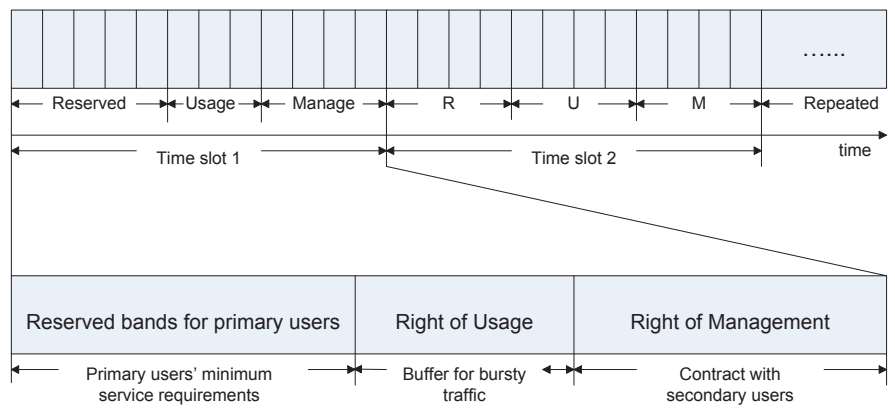

Fig. 1. Spectrum Structure.

This spectrum sharing structure is in line with PCAST report which suggests the President to open federal frequency bands for three-tier hierarchical sharing. In which, federal system have the highest priority and are protected from harmful interference. Two types of sharing exists, one with higher QoS and the other one is based on best effort.

Both spectrum usage right and spectrum management right are traded in the spectrum market as financial options through Vickrey auction. Spectrum management right is more expensive than spectrum usage right, since it has less risks and uncertainties.

\section{A. Spectrum usage right}

The spectrum usage right works as a revocable lease. PUs grant SUs the right to access the specified spectrum for a period of time. At any point during this interval, the PU can revoke the spectrum usage right; they may do so to provide higher QoS to their own users or cope with a traffic burst. They do so by sending a specified signal to the SU. In contrast to the spectrum management right, the spectrum usage right promises PUs the control over the frequency bands during the spectrum sharing. This action ensures PUs' high priority and mitigates the negative impact of spectrum sharing by allowing PUs to react promptly to variations in usage demand. Therefore, it encourages PUs to increase the spectrum supply.

SUs with $U$, on the other hand, have clear understanding that they have to terminate their service when the spectrum is recalled. This is a significant uncertainty for their operation. In order to protect the SUs' investment, a penalty function 
is implemented. The penalty is paid by PUs whenever they revoke the spectrum to the SUs whose service have been influenced. The guideline to design the penalty function is to: (1) deter PUs' abuse in revocation; (2) prevent SUs from holding the spectrum and waiting for the compensation.

\section{B. Spectrum trading as financial option}

A financial option is a derivative instrument for a future transaction on an asset, such as stock, bond, etc. The essence for option trading is to purchase the right to buy/sell the actual assets in the future. There are three components of options: premium, expiration date, and strike price. The buyer of options pay the seller a premium in order to have the right but not obligation to purchase/sell (depends on the option type) the asset at the strike price before the expiration date. The seller of the option has the obligation to sell/purchase the asset if it is requested by the buyer of the option. There are two types of financial option, call and put. Entities that estimate the asset that may decrease in value will write a call option (short call), which may require them to sell the asset at the strike price. The long call (buyer) has the right but not obligation to purchase the asset. Parties that anticipate the asset may increase in value will write an put option (short put), which means they may have to buy the asset at the strike price. The long put (buyer) has the right but not obligation to sell the asset.

The primary advantage of an option is that it helps mitigate risks and uncertainties in the future, since the buyer of options does not have the obligation to buy/sell the asset. In other words, if the value of the asset decreases, the long call will not exercise their option (ii.e., buy the asset) and their only loss is the premium and not the decrease in asset value. We adopt the option method since spectrum users also have uncertainties in the future usage of spectrum due to the nature of burst traffic and unpredictable demands. Furthermore the $U$ bands defined above behave similarly to options.

In determining spectrum contracts in $U$, PUs write an call option as [premium, spectrum cost, start time, finish time, frequency bands]. Likewise, SUs write a put option in order to buy spectrum according to the same matrix. The premium is the same as the one in financial area, and is a sunk cost for option buyer. Spectrum cost is analogous to the strike price, which determines the asset cost. Start time is the time for operation to start and it is the expiration time for the option. For example, if the SUs do not pay the spectrum cost before start time, the call option expired and PUs collect the premium. Ending time is used to determine the duration of operation. There can be more specification for spectrum other than frequency bands, such as power density, spectrum mask and so on. We choose minimum number of indicators to simplify the model.

One condition on applying options is that the underlying market should be liquid. In [8], the authors find that viable spectrum markets must have sufficient participants $(>6)$ and the spectrum supply must be roughly balanced to the demand. [9] points out that two steps are needed for market liquidity. The first one is to involve federal frequency into spectrum market. The second one is that call option should be incorporated in trading asset. In this paper, we assume multibuyer and -seller with large number of chunks of spectrum, and the market liquidity is achieved.

\section{Auction}

We implement our mechanisms in a multi-unit Vickrey (second price) auction, since it is an efficient method to enhance competition within groups and allocate the resource to whom values it the most. Bidders are ranked by their proposed spectrum price and spectrum is allocated from higher rank to lower ones. The payment is determined by the losing bids in order. For example, the winner of the first band will pay the highest losing bid. It is proven that bidders do not have incentives to manipulate in Vickrey auction.

Without losing generality, we assume that channels owned by the same PU provide the same physical characteristics (bandwidth, carrier frequency, modulation schemes, etc.), whereas channels from different PUs have various features. For example, the carrier frequency and bandwidth for TV channels are $700 \mathrm{MHz}$ and $6 \mathrm{KHz}$, and for GSM-based PUs are $900 \mathrm{MHzor} 1800 \mathrm{MHz}$ and $200 \mathrm{KHz}$, respectively [10]. Therefore, we assumes that SUs set different base value for different PUs' frequency bands, which depends on service type and technology. This value is a non-decreasing function for each individual PU's supply.

\section{Trading procedures}

The basic tenet of this framework is spectrum trading. In every spectrum trading epoch, both PUs and SUs can initiate the spectrum trading by writing an option. Options provide the flexibility to determine whether the buyer wants to start the trading or not. However, the option does not provide any flexibility after the trading begins. This problem is solved by spectrum usage right.

If SUs and PUs agree on a put option, PUs have two decisions to make: before the start time, they can choose whether to start the trading or not; during the operation, they have the opportunity to revoke the spectrum in $U$. If SUs and PUs agree on a call option, PUs do not have the choice to drop the trading if SUs exercise the option, but they can still revoke the spectrum in $U$.

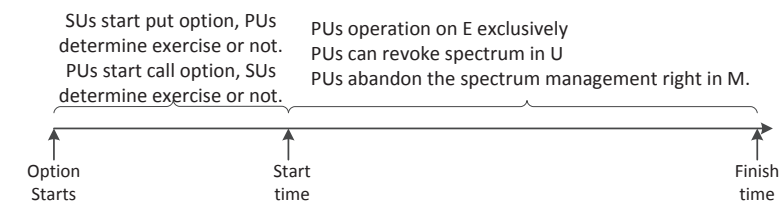

Fig. 2. Spectrum Structure.

We assume that the objective for both PUs and SUs is to maximize their utilities which will introduced in section III. The spectrum structure is not determine by PUs only. It is resulted from SUs' offer and PUs' spectrum utilization. More specifically, when SUs provide a high spectrum leasing fee, 
PUs may sell the spectrum management right even if they have demand on those bands. It is the case when PUs' service loss is less than the leasing gain.

\section{SySTEM MODEL}

\section{A. PUs' Decision in Two Cases}

We consider a heterogeneous network consisting of $n$ PUs, and $m$ SUs. In this paper, we focus on PUs' utility functions and behaviors. We assume that there are many SUs that initiate put options, each with a different spectrum demand curve. PUs compare the utility if they lease the spectrum, $U_{p}$, with the expected spectrum value without leasing, $V_{p}$, and choose the option that gives the highest profits.

We will model two cases for PUs. In the first case, PUs deterministically specify the quantity of reserved and dedicated leasing bands. The reserved band is set to satisfy minimum service requirement and the dedicated leasing bands is the idle bands. Therefore, the revenue in revocable leasing bands is superior to exogenous spectrum holes. Let $B_{p}^{R}$ equal the number of channels in the revocable leasing bands. The expected value of spectrum without leasing is calculated as $V_{p}^{R}=\theta_{p}^{R}\left(B_{p}^{R}, T_{e}\right) . T_{e}$ is the lease duration, and $\theta_{p}^{R}$ is the unit.

The expected utility of spectrum leasing in revocable spectrum bands is an optimization problem for PUs, since they can balance the potential gain with risks.

$$
\begin{array}{cc}
\text { maximize } & U_{p}^{R}(b) \\
\text { subject to } & \sum b_{s}^{r} \leq B_{p}^{R} \\
& b_{s}^{r} \geq 0
\end{array}
$$

$U_{p}^{R}(b)$ is calculated as

$$
\begin{aligned}
U_{p}^{R}(b)= & \sum_{s \in S} R_{p}^{R}\left(b_{s}^{r}, T_{e}\right)-\sum_{s \in S} \int_{0}^{T_{e}}\left(\left(P_{p}^{R}\left(b_{s}^{r}, t\right)-\right.\right. \\
& \rho_{p}^{R}\left(b_{s}^{r},\left(T_{e}-t\right)\right) \times \sigma_{p}^{R}\left(b_{s}^{r}, t\right) d t
\end{aligned}
$$

where, $S$ is the set of secondary users, $s \in S$ acquires spectrum $b_{s}^{r} \in B_{p}^{R}$ from user $p . R_{p}^{R}\left(b_{s}^{r}, T_{e}\right)$ is the revenue to be collected by PU $p$ from SU $s$ for revocable lease $b_{s}^{r}$ over time $\left[0, T_{e}\right]$. This is a non decreasing function that is determined by SUs according to their demands. When $b_{s}^{r}=0$, $R_{p}^{R}\left(b_{s}^{r}, T_{e}\right)=0 . P_{p}^{R}\left(b_{s}^{r}, t\right)$ is the penalty incurred by user $p$ for revoking $b_{s}^{r}$ at time $t . \rho_{p}^{R}\left(b_{s}^{r},\left(T_{e}-t\right)\right.$ is the extra gain provided by revocation. $\sigma_{p}^{R}\left(b_{s}^{r}, t\right)$ is the probability of revocation occurred. The second $\sum$ calculates the expected utility from revocation.

In the second case, the spectrum allocation is even more dynamic. PUs only determine the reserved bands and optimize the number of channels in revocable lease $\left(B_{p}^{R}\right)$ and dedicated lease $\left(B_{p}^{L}\right)$ based on SUs' spectrum offer and estimated risks. In other words, when SUs' offer is high, PUs will lease more dedicated bands than just idle bands. The expected value of spectrum without leasing in this case includes value from both dedicated and revocable leasing bands, $V_{p}^{a}=\theta_{p}^{a}\left(B_{p}^{a}, T_{e}\right)$.
The expected utility for leasing the spectrum for secondary usage can be optimized as

$$
\begin{array}{cc}
\text { maximize } & U_{p}\left(B_{p}^{R}, B_{p}^{L},\left(b_{s}^{r}, b_{s}^{l}\right)_{s \in S}, T_{e}\right) \\
\text { subject to } & b_{s}^{i} \geq 0, i=r, l \\
& \sum_{s \in S} b_{s}^{r} \leq B_{p}^{R} \\
& \sum_{s \in S} b_{s}^{l} \leq B_{p}^{L} \\
& B_{p}^{R}+B_{p}^{L}=B_{p}^{a}
\end{array}
$$

$U_{p}\left(B_{p}^{R}, B_{p}^{L},\left(b_{s}^{r}, b_{s}^{l}\right)_{s \in S}, T_{e}\right)$ is the utility for PU $p$ in the second case. It is depends on the total number of available spectrum $\left(B_{p}^{a}=B_{p}^{R}+B_{p}^{L}\right)$ and its allocation, SUs' demand quantity $\left(b_{s}^{r}, b_{s}^{l}\right)$ and proposed prices.

$$
\begin{aligned}
U_{p} & =\sum_{s \in S} R_{p}^{L}\left(b_{s}^{l}, T_{e}\right)-\sum_{s \in S} \rho_{p}^{L}\left(b_{s}^{l}, T_{e}\right)+\sum_{s \in S} R_{p}^{R}\left(b_{s}^{r}, T_{e}\right) \\
& -\sum_{s \in S} \int_{0}^{T_{e}}\left(P_{p}^{R}\left(b_{s}^{r}, t\right)-\rho_{p}^{R}\left(b_{s}^{r},\left(T_{e}-t\right)\right) \sigma_{p}^{R}\left(b_{s}^{r}, t\right) d t\right.
\end{aligned}
$$

where, $b_{s}^{i}$ is the number of channels that SU $s$ bought from revocable lease $r$ or dedicated lease $l . R_{p}^{L}\left(b_{s}^{l}, T_{e}\right)$ is the revenue collected by PU $P$ from SU $s$ for dedicated leasing band $b_{s}^{l}$ over time $T_{e}$. Since PUs cannot revoke spectrum in the dedicated leasing band, they will lose the potential revenue if there is demand. $\rho_{p}^{L}\left(b_{s}^{l}, T_{e}\right)$ captures the potential demand for PUs in the dedicated leasing band.

\section{B. Risk Function}

The risk function reflects PUs' estimate of potential service demand. We use three types of distributions to model the risk. The first risk function follows uniform distribution, which means the probability potential demand is the same at any point of time.

$$
R 1=\frac{1}{T e}
$$

The second risk function is an exponential distribution. From the PUs' perspective, it means that the probability of service demand decreases with time. In other words, if PUs do not have service demand now, it is less likely that they will need the spectrum in the near future.

$$
R 2=\lambda e^{-t \lambda}
$$

The logic behind the third risk function is that the probability of service demand will increase with time. This line captures the assumption that the longer the duration, the more likely that there will be service demand.

$$
R 3=1-\lambda e^{-t \lambda}
$$

\section{Penalty Function}

For the sake of brevity, we only consider two types of penalty functions to demonstrate the impact of penalty functions on a PUs' decision and utility. There are certainly more intermediate or more appropriate penalty function for different services. The variable in penalty function is the revocation time 
$t$. The first type of penalty function is a straight line, where we assume that the spectrum utility increase with time linearly.

$$
P 1=\frac{\sum_{s \in S} R_{p}^{R}\left(b_{s}^{r}, T_{e}\right) \times t}{T e}
$$

The reason behind the second type of penalty function is that since SUs have already use some spectrum, PUs will pay less than the value of the spectrum.

$$
P 2=\left(1+\sum_{s \in S} R_{p}^{R}\left(b_{s}^{r}, T_{e}\right)\right)^{t / T e}-1
$$

\section{Evaluation}

In this section, we evaluate the profits that PUs can gain in different situations. Three major factors affect the PU's decision: spectrum lease duration, penalty function, and the PUs' estimated spectrum gain based on their own wireless services.

\section{A. PUs' choices in static situation}

Fig. 3 shows the PUs' penalties in different risk and penalty combinations when $T e=2$. pirj indicates the combination of risk and penalty functions. For example, $p 1 r 1$ means PUs' estimate of their potential service demand is type $R 1$ and the penalty that $\mathrm{s} / \mathrm{he}$ will pay is defined by the penalty function $P 1$. From this figure we can see that $\mathrm{P} 2$ gives less penalty than $\mathrm{P} 1 . \mathrm{R} 2$ gives the smallest penalty since the risk of revoking spectrum decreases with time. R3 gives the highest penalty since both the risk and the penalty increase with time. This figure provides guidance for PUs and SUs to negotiate the penalty function. The penalty value varies significantly even with the same risk function. Moreover, accurately estimating the risk function is important for PUs. In addition, the penalty enlarges when $T e$ increases, which is shown in Fig.4. Thus, PUs should shorten the spectrum lease duration when they cannot foresee the future usage accurately.

The purpose of the second simulation is to show whether adding spectrum usage rights can bring extra profits in a static situation, where PUs determine the number of leaseable channels. In Fig.4, we capture the largest profits for PUs under 18 situations with different combination of risk and penalty functions when $T e=2,4,5$ respectively. We assume that there are 3 channels that have potential usage and can be revocably leased. The number on each bar shows the number of channels that lead to the largest profits. It is clear that when penalty is low, PUs' profits increase with Te. For example, profits in $\mathrm{p} 1 \mathrm{r} 2$ and $\mathrm{p} 2 \mathrm{r} 2$ are always higher than $\mathrm{p} 1 \mathrm{r} 1$ and $\mathrm{p} 2 \mathrm{r} 1$. However, when the penalty is high, as is the case with p1r3 and p2r3, the PU's profit decreases with lease duration. In some cases, even selling one channel under revocable leasing results in negative profits. Therefore, there is no profit to leasing spectrum usage rights in these situations. PUs could choose to reduce the spectrum trading period or only lease the idle bands.

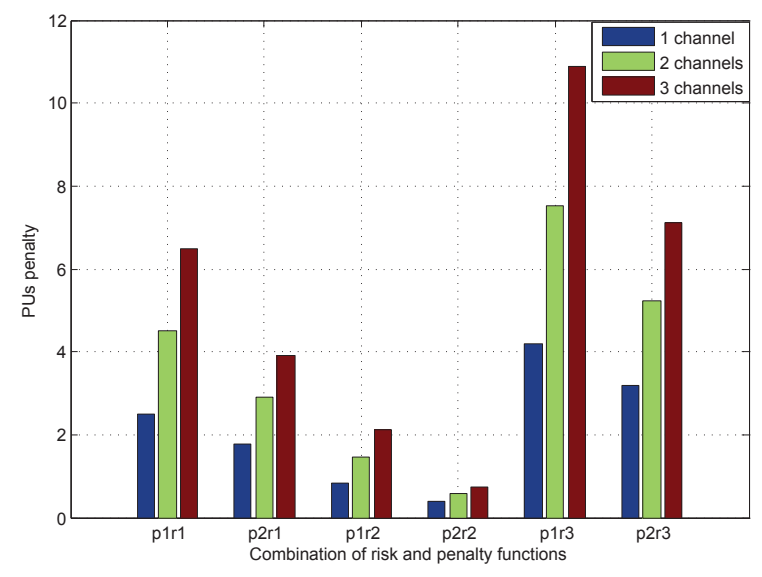

Fig. 3. PUs' penalty in static assignment.

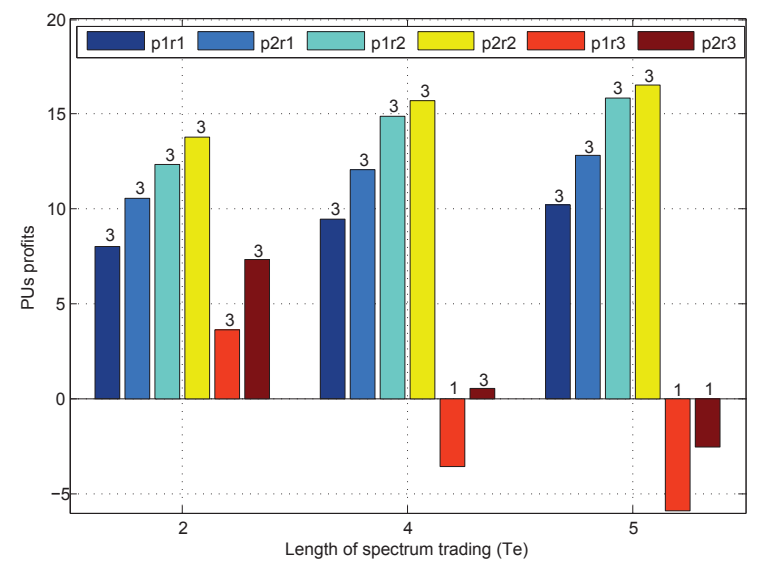

Fig. 4. PUs' profits in static assignment.

\section{B. PUs' choices in dynamic situation}

In this simulation, we assume that there are 3 idle bands and 5 channels have potential usage. PUs dynamically determine the number of channels under dedicated and revocable lease terms to maximize their profits. Fig.5 provides the leasing strategy that results in the highest profit in each situation. The first digit above each bar is the number of channels in the dedicated leasing bands, and the second on is the number of channels in the revocable lease. As shown by the $x$ axis label, there are five groups with different $T e$ and $E g$. Te is the lease duration, $E g$ is PUs potential gain if they do not lease the spectrum. The expected $E g$ is time dependent since the wireless service gain is partially related to service time. Therefore, $E g$ is proportional to $T e$.

In Fig.5 we can see that, within each group, it is more profitable for PUs to lease more revocable bands when the penalty is low. The reason is that if the penalty is low, PUs prefer to reserve the bands for their own usage. When the penalty is higher than the gain from their own services, they would rather lease them in dedicated bands for higher spectrum leasing fees.

Next, we compare the strategy and profits across groups. The first three groups assume the same potential profits and 
vary in the lease duration. It is clear that PUs are more aggressive when lease duration is low, since PUs can estimate the potential usage more precisely. The estimated potential usage also impacts the PUs' strategy and profits. Let us compare groups $1,4,5$, where $T e=10$ and $E g$ varies. It is rational for PUs to be more aggressive in leasing spectrum in dedicated bands when the potential gain from their own services is low. In summary, PUs' strategy and profits are highly situational: they vary with lease duration, potential gain from their own services, as well as risk and penalty functions.

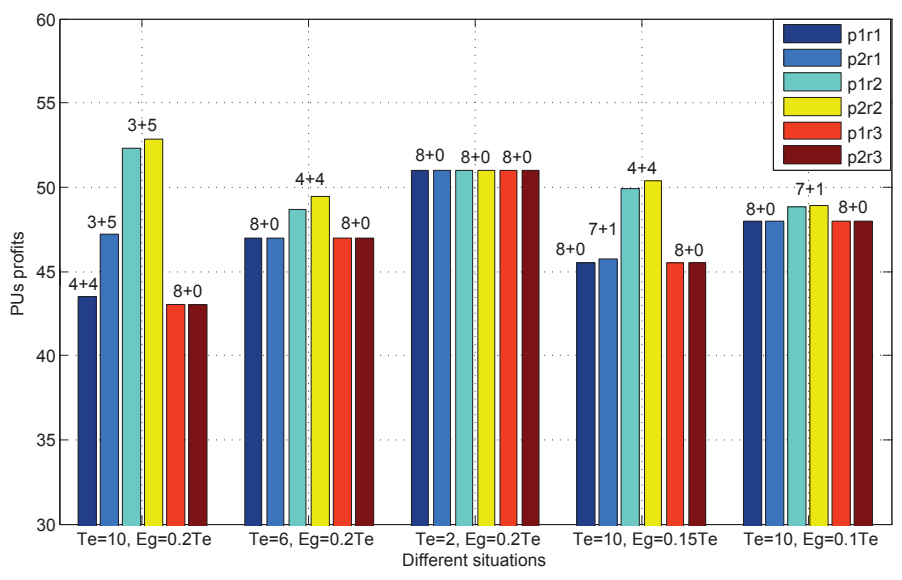

Fig. 5. PUs' profits in dynamic assignment.

\section{Impacts from options}

In the above sections, we analyze the profits that PUs can earn when the trading is successful. As we mentioned before, spectrum trading may not be successful every time, since the option buyer does not have the obligation to buy/sell the asset. Therefore, the expected utility for PUs also depends on the option type. More specifically, the expected utility for PUs in a call option is $U_{\text {call }}=(1-\alpha)$ Pre $+\alpha \times U_{p}$. Where, Pre is the premium collected by PUs, and $\alpha$ is the rate of successful trading. PUs pay a premium in a put option, thus the expected utility for PUs in a put option is $U_{\text {put }}=(1-\alpha)(-$ Pre $)+$ $\alpha \times U_{p}$.

In Fig.6, we assume the optimum profits 43.5, which we determined above under the assumption of dynamic trading with penalty type 1 and risk type 1 and $T e=10, E g=0.2 T e$. The first letter in the legend indicates the option type $(c$ is call option and $p$ is put option). The percentage indicates the amount of premium. For example, $1 \%$ means the premium is one percent of the profits, which is 0.435 . It is clear that call options provide higher expected utility since PUs collect the premium. When the premium is low, the expected utility in both options are almost the same. Moreover, the expected utility increase with the successful trading rate. When the trading is one hundred percent successful, the expected utility equals to PUs' profits.

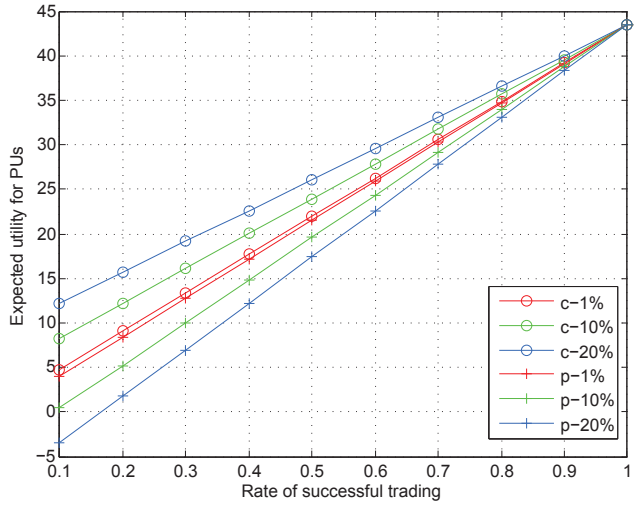

Fig. 6. PUs' expected utility impacted by options.

\section{CONCLUSION}

In this paper, we proposed a coopetition framework which boost competition within PUs and SUs and encourage cooperation between groups to create more endogenous spectrum holes. We suggest two types of trading: revocable and dedicated leasing. In revocable leasing, PUs can retrieve spectrum back at any point of time with a penalty. PUs cannot touch the spectrum in dedicated leasing, so the price is higher in return. With this mechanism, we encourage PUs to lease more spectrum and protect SUs' investment. We show PUs' profits in different combination of penalty and risk functions, as well as PUs' estimated service gain. We identified the factors that impact profits and also pointed out situations that leads to the largest profits. In the future research, we will analyze the profits and strategies for SUs. Then, we will implement our mechanisms in agent-based model to capture the interactions between PUs and SUs.

\section{REFERENCES}

[1] F. Force, "Report of the spectrum efficiency working group," ET Docket, no. 03-237, 2002.

[2] M. B. Weiss, "Spatio-temporal spectrum holes and the secondary user," in IEEE DySPAN. DySPAN: IEEE, May 2011, pp. 1-7.

[3] Z. Ji and K. Liu, "Multi-stage pricing game for collusion-resistant dynamic spectrum allocation," Selected Areas in Communications, IEEE Journal on, vol. 26, no. 1, pp. 182-191, 2008.

[4] D. Niyato and E. Hossain, "Competitive spectrum sharing in cognitive radio networks: a dynamic game approach," Wireless Communications, IEEE Transactions on, vol. 7, no. 7, pp. 2651-2660, July 2008.

[5] Y. Tan, S. Sengupta, and K. Subbalakshmi, "Competitive spectrum trading in dynamic spectrum access markets: A price war," in Global Telecommunications Conference, 2010. GLOBECOM '10. IEEE, Dec. 2010, pp. $1-5$.

[6] J. Huang, R. A. Berry, and M. L. Honig, "Auction-based spectrum sharing," Mob. Netw. Appl., vol. 11, no. 3, pp. 405-418, Jun. 2006

[7] S. Gandhi et al., "A general framework for wireless spectrum auctions," in New Frontiers in Dynamic Spectrum Access Networks, 2007. DySPAN 2007. 2007 2nd IEEE International Symposium on, April 2007, pp. 2233

[8] C. E. Caicedo and M. B. Weiss, "The viability of spectrum trading markets," IEEE Communications Magazine, vol. 49, no. 3, pp. 46-52, March 2011.

[9] M. Bykowsky, "A secondary market for the trading of spectrum: promoting market liquidity," Telecommunications Policy, vol. 27, no. 7, pp. 533-541, 2003

[10] L. Gao, Y. Xu, and X. Wang, "Map: Multiauctioneer progressive auction for dynamic spectrum access," Mobile Computing, IEEE Transactions on, vol. 10, no. 8, pp. $1144-1161$, Aug. 2011. 